\title{
A Summary of the Homogeneous 5D Universe Creation Model: Expressed in the Dirac Second-Order Quantization Representation
}

\author{
Kai Wai Wong1, Wan Ki Chow ${ }^{2}$ \\ ${ }^{1}$ Department of Physics and Astronomy, University of Kansas, Lawrence, USA \\ ${ }^{2}$ Department of Building Services Engineering, The Hong Kong Polytechnic University, Hong Kong, China \\ Email:kww88ng@gmail.com
}

How to cite this paper: Wong, K.W. and Chow, W.K. (2021) A Summary of the Homogeneous 5D Universe Creation Model: Expressed in the Dirac Second-Order Quantization Representation. Journal of Modern Physics, 12, 123-138.

https://doi.org/10.4236/jmp.2021.123012

Received: December 22, 2020

Accepted: February 5, 2021

Published: February 8, 2021

Copyright $\odot 2021$ by author(s) and Scientific Research Publishing Inc. This work is licensed under the Creative Commons Attribution International License (CC BY 4.0).

http://creativecommons.org/licenses/by/4.0/

\begin{abstract}
A summary of the homogeneous 5D universe model is expressed in the Dirac second-order quantized representation for the magnetic monopoles, identified in terms of the Higgs Bosons, and through systematic ordered excitations of the Higgs vacuum obtained the non-homogeneous 4D Lorentz manifolds filled with masses, corresponding to making space dimension reduction projections, and thus realization of Newtonian gravity, followed by the 3D space symmetry breaking into $2 \mathrm{D} \times 1 \mathrm{D}$ that produces the Perelmann-entropy and Ricci-Flow mappings, resulting in the realization of Poincare spheres, represented by nucleus such as Carbon 12, all the way to stars, and matter filled discs, such as stars in galaxies and 2D carbon-based molecular structures like nitrogenous bases. Finally, the forming of RNAs and DNAs, then life forms.
\end{abstract}

\section{Keywords}

Magnetic Monopoles, Higgs Bosons, Space Reduction Projections, Perelmann Mappings, Nitrogenous Bases and Life Forms

\section{Introduction}

The 4D inhomogeneous Lorentz manifold was obtained by a space dimension projection from a 5D homogeneous space-time manifold [1]. This topological projection is irreversible and is shown to be mathematically equivalent to the Perelmann-entropy Ricci-flow mappings [2] [3] in deriving the donut and the spherical 4D Lorentz mass distributions [4]. It was further shown via the dimension reduction projections, the $5 \mathrm{D}$ manifold must become a $4 \mathrm{D}$-Lorentz mani- 
fold with direct products to the superposition of the Semi-simple Compact Lie Groups SU(2) + SU(3), as the McGlinn theorem [5] must be obeyed. This topological result is in complete agreement to the Gell-Mann standard model [6]. It was then further shown that by applying Lorentz gauge invariance, in conjunction with deriving the Gluon fields as the tensor products of the 4 Electromagnetic vector potentials, the hadron masses can be very accurately calculated [1]. On top of that achievement, the neutron oscillation was also obtained [7]. As the 4 electromagnetic vector potentials satisfy the $4 \mathrm{D}$ homogeneous Klein-Gordon differential equation, the 5D homogeneous 2 nd-order differential equation must generate 5 vector potentials instead of just 4 . This extra component of the solution was originally proposed by Maxwell [8] and was coined as the magnetic monopole potential. It was relatively recent that we have derived its explicit form [9]. It was then possible to derive the explicit monopole unit as $+2 \mathrm{ec}$ and $-2 \mathrm{ec}$. Where they were the product from two oppositely charged, opposite momenta along the $3 \mathrm{D}$ spherical radius, and massless spinor solutions to the Dirac linearized 5D equation. Because of the opposite momenta of the spinor pair, this magnetic monopole magnitude must be carried by two Diagonal Long Range Ordered Bosons with opposite magnitudes. Since each charged massless spinor like a neutrino has quantized momentum and energy, these massless charge neutral Bosons also carry energy, despite being in the Bose-Einstein condensed state. It is this mathematical feature that makes them equivalent to the Higgs Bosons [10]. And they must completely fill the homogeneous 5D manifold. Hence on recognizing the universe as a Big Bang, started with absolutely nothing, that is no time and no space, the homogeneous 5D universe must be given by a Higgs vacuum. From which, the creation of mass through space projections or the equivalent Perelmann mappings must be equivalent to the excitation of this Higgs vacuum. In fact, it was discovered by the CERN supercollider experiment that a $125 \mathrm{GeV}$ proton-proton resonance exists [11] and thus verified both the Higgs theory as well as the projection theory [1]. It is through all of these rigorous mathematics and experimental results that we believe it might be helpful to represent these monopole-Higgs Bosons in the Dirac second quantized representation, so that we can further clearly show why there is only energy with no net charge, and net monopoles, actually existed in the homogeneous 5D universe starting at the beginning in complete agreement to the Big Bang model, and the Higgs vacuum. What we have not yet been able to explain after 4D Lorentz manifolds were created, that the dynamics of the masses within would indeed lead to Einstein's General Relativity [12], with the Gravity tensor equation [13], where Einstein postulated the existence of a Cosmic constant. And if it is in some way mathematically related and obtained by excitation giving rise to the symmetry breaking of the Higgs bosons via either the breaking of their Diagonal Long Range Order (DLRO) or the explicit breaking of the charge neutrality, and therefore the Bosons itself, such that a charge density distribution term to the Gravity tensor equation can be realized and the Einstein Cosmic constant ex- 
plained. And how the truncation on the bosons energy range together with choice of remaining 3D space symmetry can result in the natural forming of molecules that have natural excitation spectra, that can be divided in turns of coherent and de-coherent [14], a very essential philosophical point raised by Schrodinger [15]. And lastly at the lowest frequencies how it is correlated to the life forms' DNA spectra [1] [16]. It is these loose ends to the 5D grand unified field theory [1] our intension to attempt address in this paper.

\section{The Magnetic Monopoles and the Higgs Bosons}

According to the 5D homogeneous manifold, there must exist 5 vector potentials, represented by the 4 electromagnetic vector potentials and an orthogonal 5th Maxwell magnetic monopole potential as proposed by Maxwell [8]. The explicit form of this monopole potential was recently derived by us [9], with the magnetic monopole strength given by a DLRO Boson field composed of the product from two opposite momentum massless [1], but with e and -e charges spinor pair, such that the monopole strengths are given by

$$
M(+)=2 \mathrm{ec} ; \text { and } M(-)=-2 \mathrm{ec} .
$$

Let us denote in terms of Dirac second quantization representation.

Let $c_{\vec{p}}$ be that of the destructive operator for the charge "e" e-trino with momentum $\vec{p}$; and $d_{-\vec{p}}$ be that of the destructive operator for the charge "-e" anti-e-trino, and with spin indices suppressed. Because e-trino and anti-e-trino are massless spinor, despite their name we gave, they are not the conjugate of each other, massless spinor does not have corresponding anti-spinor, hence $d_{-\bar{p}}$ and $c_{\vec{p}}$ commutes, such that an opposite momentum pair is a Boson with net 0 momentum and forms a Diagonal Long Range order, with a current magnitude of either $2 \mathrm{ec}$, or $-2 \mathrm{ec}$, which gives the magnetic monopole strengths. An opposite momentum pair of the same spinor, say e-trino, forms an off-diagonal long range order Boson but carries net 0 monopole strength. All massless fields carry momentum of $h v / c$, with energy $h v$. A DLRO e-trino and anti-e-trino pair thereby carries net 0 momentum and 0 center of frame energy. It does not mean this DLRO pair has no energy. In fact, it has a total energy E of $2 \mathrm{hv}$. Thus such an e-trino and anti-e-trino DLRO pair Boson field must be in the Bose-Einstein condensed state. Unlike the photon, as when its momentum is 0 , so is its energy. Thus the photon cannot exist in the Higgs vacuum, and therefore cannot exist in the homogeneous 5D manifold. In another word, inside the 5D domain, we cannot have photons. To some this can be viewed as a "black hole" region. Except it does not mean photons are sucked into this 5D domain! And definitely not equivalent to the concept of a black hole, where matters are sucked in and reverted back to pure energy. On the contrary, it verifies Maxwell's monopole theory, that there exists a 5th magnetic monopole potential orthogonal to the 4 electromagnetic vector potentials, such that it must be a potential along the Fermat's amplitude, resembling the Coulomb potential of a point charge. Hence, one can conclude that the electromagnetic fields actually serve as a boundary of 
the 5D manifold where the monopoles, or the Higgs Bosons exist.

Because the homogeneous 5D manifold does not have time reversal symmetry, the creation of matter through the excitation of the Higgs vacuum constitutes an irreversible entropy mapping, the matter then created must then be in an inhomogeneous 4D Lorentz manifold, as demonstrated by the Perelmann Ricci-flow mapping. Such that no reverse mapping process is allowed. In short matter cannot be reverted back to pure energy and the Higgs vacuum completely restored. Meaning the concept of a "black hole" as such an infinite sink of matter cannot exist in the 5D universe, irrespective of gravity [17] [18].

Therefore the ensemble magnitude of $\langle M(+) M(-)\rangle$ are given in quantum term as

$$
\langle M(+)\rangle=2 \mathrm{ec} N_{E}(+) ;\langle M(-)\rangle=-2 \mathrm{ec} N_{E}(-)
$$

where $N_{E}(+)=\left\langle B_{E}^{*}(0) B_{E}(0)\right\rangle ; N_{E}(-)=\left\langle D_{E}^{*}(0) D_{E}(0)\right\rangle$ such that $B_{E}(0)=c_{\bar{p}} d_{-\bar{p}}$ and $D_{E}(0)=d_{\bar{p}} c_{-\bar{p}}$, and \langle\rangle represents the canonical ensemble average.

As these boson fields are in the Bose-Einstein condensed state, they must be the Higgs fields and populate the Higgs vacuum.

\section{Properties of the Higgs Bosons}

To better understand the $B_{E}(0)$ and $D_{E}(0)$ topologies, let us illustrate in Figure 1 with color vectors: $c_{\bar{p}}$ as a red vector along $+\hat{r}$, from the origin of the 5D manifold, and $d_{-\bar{p}}$ then as a blue vector also along $+\hat{r}$. Then $B_{E}(0)$ would carry a current of $2 \mathrm{ec}$ along $+\hat{r}$. While $D_{E}(0)$ carries a current of $-2 \mathrm{ec}$ also along $+\hat{r}$.

Now let us illustrate in Figure 2 the operations of $\tilde{\boldsymbol{C}}$ Charge and $\tilde{\boldsymbol{P}}$ Parity acting on $B_{E}(0)$ and $D_{E}(0)$.

It is obvious $\tilde{\boldsymbol{C}} B_{E}(0)$ changes the $+\hat{r}$ current to $-2 \mathrm{ec}$. In another word $\tilde{\boldsymbol{C}} B_{E}(0)=D_{E}(0)$

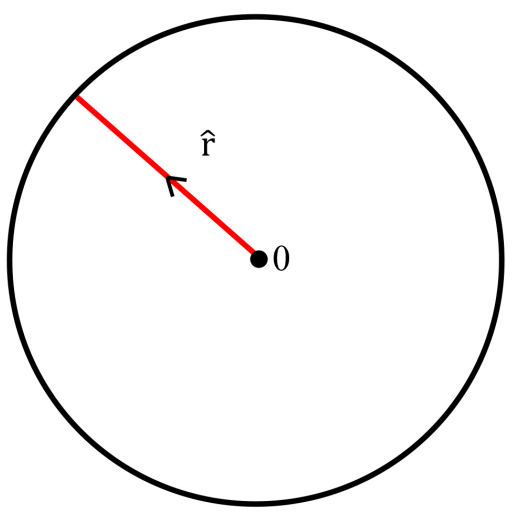

(a)

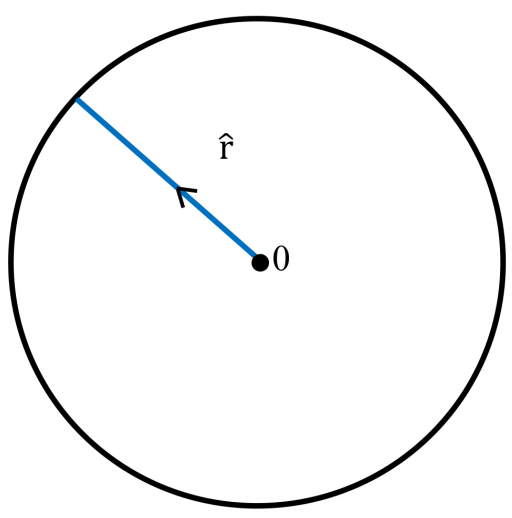

(b)

Figure 1. Illustration of $B_{E}(0)$ and $D_{E}(0)$. (a) The red line vector from 0 , the center represents the Boson $B_{E}(0)$. (b) The blue line represents the $D_{E}(0)$. 


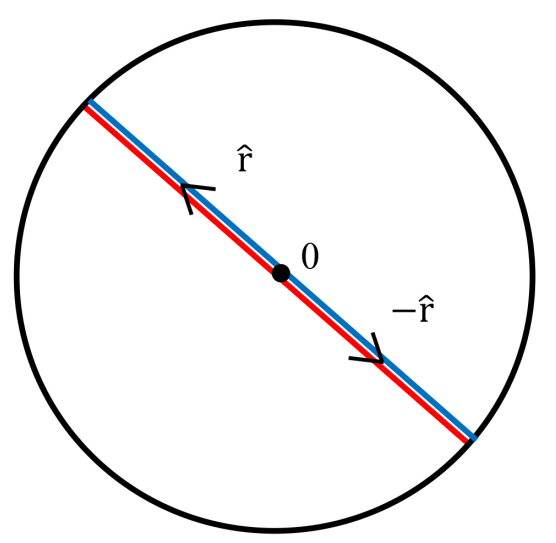

Figure 2. Illustration of Parity operation on $B_{E}(0)$ and $D_{E}(0)$. The red and blue parallel lines across the circle diameter represent $B$ $+D$ and its image $B^{\prime}+D^{\prime}$ due to $\tilde{\boldsymbol{C}} \tilde{\boldsymbol{P}} \tilde{C}$.

The parity $\tilde{\boldsymbol{P}}$ is a mirror image operation. Hence it changes from $+\hat{\boldsymbol{r}}$, to $-\hat{r}$ from the manifold center. Thus $\tilde{\boldsymbol{P}} B_{E}(0)$ represents $B_{E}^{\prime}(0)$ with $-\hat{r}$ rather than $D_{E}(0)$. The sum of $B_{E}(0)$ and $B_{E}^{\prime}(0)$ results in 0 magnetic monopole strength.

Now let us consider the operation $\tilde{\boldsymbol{C}} \tilde{\boldsymbol{P}} \tilde{\boldsymbol{C}}$ on $B_{E}(0)$. We have $\tilde{\boldsymbol{C}} \tilde{\boldsymbol{P}} \tilde{\boldsymbol{C}} B_{E}(0)=\tilde{\boldsymbol{C}} \tilde{\boldsymbol{P}} D_{E}(0)=\tilde{\boldsymbol{C}} D_{E}^{\prime}(0)=B_{E}^{\prime}(0)$. Which looks like $D_{E}(0)$ but actually is the parity image of $B_{E}(0)$, a distinct state in the 5D manifold. Hence starting with any DLRO monopole Boson, $\tilde{\boldsymbol{C}} \tilde{\boldsymbol{P}} \tilde{\boldsymbol{C}}$ acting successively on it will produce the remaining 3 independent of the 4 DLRO Bosons. Since the sum of $B_{E}(0)+B_{E}^{\prime}(0)$ is equal to having $M(+)+M(-)=0$. Similarly $D_{E}(0)+D_{E}^{\prime}(0)$ also resulted in no net magnetic monopole strength. It is this result that shows clearly that in the $5 \mathrm{D}$ homogeneous manifold we preserve net 0 magnetic monopole strength, as well as net 0 charges. Hence all that remains in the $5 \mathrm{D}$ manifold is energy carried by the $B_{E}(0)$ and $D_{E}(0)$ fields. Thus together with uncertainty principle, illustrates how the creation of the homogeneous $5 \mathrm{D}$ universe, must give rise to the Universe's Big Bang. And all the energies are carried by the $B_{E}(0)$ and $D_{E}(0)$ bosons uniformly covering the $5 \mathrm{D}$ as it expands with time, thus making up the Higgs vacuum.

It should be noted that matter need not start being created at the same instant as the creation of the homogeneous 5D space-time. In mathematics, only infinity and zero can be divided as many times as we like, and this divided set all remain infinite and zero each. Thus the Perelmann-Ricci-flow entropy mappings for the creation of galaxies by exciting the Higgs vacuum can happen later and sequentially, leaving galaxies well separated.

\section{Excitation of the Higgs Vacuum and Mass Creation Example}

The $5 \mathrm{D}$ vacuum is filled with $B_{E}(0)$ and $D_{E}(0)$ bosons in the Bose-Einstein condensed state. An excitation requires the breaking of the net 0 momentum of 
these boson fields. We can do that as an example by changing the $c_{\bar{p}} d_{-\bar{p}}$ pair for the $B_{E}(0)$ field to $c_{\bar{p}+\bar{q}} d_{-\bar{p}+\bar{s}}$, where the additional momentum $\vec{q}$ and $\vec{s}$ need not be along $\hat{r}$. This new pair now has a net center of frame momentum of $\vec{q}+\vec{s}$. Hence it gives rise to a nonzero excitation energy $E^{\prime}=c|\vec{q}+\vec{s}|$, and hence changes the Bose-Einstein distribution for the $\mathrm{B}$ or $\mathrm{D}$ field to

$$
\frac{1}{\mathrm{e}^{E^{\prime} / k T}-1}
$$

As long as $E^{\prime}>0$, the Bose distribution can be expanded in a convergent infinite power series, of $\mathrm{e}^{-C j}$, where $C=E^{\prime} / k T$, and $j=1,2,3, \cdots$. [19]. Thus for $E^{\prime} \geq m(e) c^{2}$, the rest energy of an electron, with high enough $T$, that of Bethe fusion temperature, matter will be created as suggested by Higgs theory [10]. Topologically it must correspond to destroying the 5D homogeneity. Hence is equivalent to creating a 4D Lorentz manifold boundary on the 5D manifold. As an illustration, let us take $\vec{q}=\vec{s}$ and perpendicular to $\hat{r}$, we will obtain a net angular momentum $2 \vec{q} x \vec{r}_{o}$ around 0 . Where $\vec{r}_{o}$ is the time frozen core radius perpendicular to $\vec{q}$. Thus this excited angular momentum must be balanced by an equal but opposite angular momentum from the rotating masses outside the 5D domain and in the enclosing 4D Lorentz manifold. While if $\vec{q}=-\vec{s}$, we would result in a creation of a dipole magnetic field, as $\vec{q}$ generates a current loop with radius $r_{o}$. It is these excitations that correspond to performing the Perelmann entropy mappings [2] [3]. By separating the results from the Perelmann mappings into preserving the $3 \mathrm{D}$ space symmetry, we obtain the Poincare sphere, starting in the microscopic scale for the leptons to the nucleus with a time frozen 5D core, such as Carbon 12, to the astronomical scale stars and planets. Hence for an isolated spherical time frozen core, the remainder $B_{E}(0)$ and $D_{E}(0)$ fields within must be standing waves, with frequencies obeying $v=n c / 4 r_{o}$, where $r_{o}$ is the core radius, and $n=1,2,3, \cdots$. Hence the allowed eigen energy $\mathrm{E}$ for $B_{E}(0)$ and $D_{E}(0)$ bosons form a discrete set. This implies the smaller the core radius the higher the excitation energy is permitted as long as it is less than $h v / 4 r_{o}$. Or in a disc like structure, as corresponding to the Ricci-flow mapping, thus breaking $3 \mathrm{D}$ into $2 \mathrm{D} \times 1 \mathrm{D}$, such as represented by the $\mathrm{SU}(3)$ generators in space to space conformal projection, to that astronomical scale the galactic structure. With the standing wave restriction applies to the $2 \mathrm{D}$ circle only. In fact, this broken 3D space symmetry is represented by SU(3) in charge separation between $-\mathrm{e}$ and $+\mathrm{e}$ obeying gauge invariance. Hence producing current source that in turn produces the 4 EM potentials. And for a completely enclosed time frozen 5D void core, it can be elliptical and not solely spherical. It is also very reviewing to investigate the amount of total number of Bose fields $\mathrm{N}$ excited due to Perelmann mappings, and the total energy $\left\langle E^{\prime}\right\rangle$ used as a function of Temperature $T$ and the frozen time $t_{o}$ as measured from each sub-5D manifold by integrating $E^{\prime}$ from the lower cut-off energy $E_{o}$, with the 3D spherical symmetry, and the number $n$ of new Bosons those of atoms in the periodic table created. We obtain 


$$
\begin{gathered}
N=\frac{\frac{n 4 \pi}{3}\left(c t_{o}\right)^{3}}{V k T} \sum_{j=1}^{\infty} \frac{\mathrm{e}^{-j E_{o} / k T}}{j} \\
E^{\prime}=\frac{n 4 \pi}{3 V}\left(c t_{o}\right)^{3} \sum_{j=1}^{\infty}\left\{E_{o}-\frac{k T}{j}\right\} \frac{\mathrm{e}^{-j E_{o} / k T}}{j}
\end{gathered}
$$

where $V$ is the volume of the Lorentz space.

$N$ varies with $t_{o}^{3}, n$ and inversely with $T$. Thus the lower $T$ value we have more matter including complex matter structures created.

While from $\left\langle E^{\prime}\right\rangle$ always positve, $E_{o}$ must be greater than $(k T) / j$. Meaning $E_{o}$ is discretized as $T$ is fixed. It is this result that created the different stages of creation. The lower the Temperature $T$, the lower would be the allowed excited spectrum of the B, D bosons. For the initial matter creation at Bethe Fusion temperature, $n=2$, due to only $\mathrm{B}$ and $\mathrm{D}$ bosons. $E_{o}$ should be that of the proton pure Quark rest mass, then the highest $j$ value is roughly 64 as the electron rest mass is roughly 1/64 that of the Quark's, with the neutron in between.

As $T$ decreases, $t_{o}$ increases, and $E_{o}$ decreases as it represents the gluon binding, and with the atomic Coulomb binding energy, with the number $\mathrm{n}$ being those of atoms, from hydrogen to the heaviest element. Thus $\mathrm{n}$ becomes quite large. On the other hand, Heavy hydrogen with an extra neutron is not a Boson, and does not satisfy Equation (3). $E_{o}$ becomes the molecular binding energy cut-off. Leading to the coherent and de-coherent series for molecules studied by Schrodinger, Geesink et al. and many other physicists.

As masses within the 4D Lorentz manifold move, we need to express this Lorentz manifold in a covariant form. Hence it gives us naturally the Covariant Riemannian curvatures, which would give us the law of gravity for matter [12], but with a naturally build-in time frozen $5 \mathrm{D}$ core, a repulsive worm hole [20] filled with repulsive e-trinos and anti-e-trinos [21].

Following General Relativity [12], we obtain the 4D gravity equation for a mass $M$ within a covariant $4 \mathrm{D}$ Lorentz manifold

$$
G_{\mu v}=8 \pi G\left(T_{\mu v}-\rho\left(E_{o} / k T, \vec{r}\right) g_{\mu v}\right)
$$

where $G_{\mu v}$ is the $4 \times 4$ gravity tensor, $G$ is the Newtonian constant, $T_{\mu \nu}$ is the $4 \times 4$ energy tensor, while $g_{\mu v}=\frac{\partial x_{\mu}}{\partial x_{v}}$ and $\rho\left(E_{o} / k T, \vec{r}\right)$ represents the energy density of the e-trino, anti-e-trino which is geometry and relative phase dependent. For example, if the e-trino and anti-e-trino rotate in phase with a radius $r_{o}$, or in opposite phase, these geometrical restrictions lead to self-rotation of stars and planets as well as a dipolar magnetic field [22] [23]. And then there is the totally incoherent separated e-trino, anti-e-trino distribution in a non-regular $4 \mathrm{D}$ geometry

$$
\rho\left(E_{f} / k T, \vec{r}\right)=\left\langle c|\vec{p}| c_{\vec{p}}^{*} c_{\vec{p}} \mathrm{e}^{i \vec{p} \cdot \bar{r}}\right\rangle_{f}+\left\langle c|\vec{p}| d_{\vec{p}}^{*} d_{\vec{p}} \mathrm{e}^{i \vec{p} \cdot \vec{r}}\right\rangle_{f}
$$

where \langle\rangle$_{f}$ represents the Fermi average, and $c_{\vec{p}}, d_{\vec{p}}$ are massive electrons 
and protons. Only through this would we obtain an electromagnetic component of field energy.

Note because mass is created from the excitation of the Higgs vacuum $\rho$ is not due to the B.E. Bosons B and D.

Should we define Einstein cosmological constant

$$
\Lambda=8 \pi G \rho\left(E_{o} / k T, \vec{r}\right)
$$

we observed it behaves like the Wheeler wormhole [20]. For the case given by Equation (6) and $E_{f} / k T \rightarrow 0, \rho(\vec{r} \rightarrow 0) \rightarrow 0$. The gravity Equation (5) leads to a singular solution resembling a "black hole". Except as masses spiral into $\vec{r} \rightarrow 0, E_{o} / k T$ will increase and the singularity will be removed.

It is not necessary to apply the Einstein-Kaluza-Klein compacting of the proper time to unify with electromagnetic theory, as the homogeneous Maxwell $4 \mathrm{D}$ is a natural boundary of the homogeneous $5 \mathrm{D}$ manifold and thus will coexist with the Lorentz 4D manifold.

Going down in the $\mathrm{C}$ cut-off value after protons, neutrons and electrons were created, we would obtain the periodic table elements, so that as the universe expands, creation of galaxies, stars and planets formation follows [22] [23]. Resulting in stars and planets having self-rotations and a dipolar magnetic field. Finally on the climatic surface of a planet allows further breaks of the 3 dimension space symmetry into $2 \mathrm{D} \times 1 \mathrm{D}$, as gravity defines the normal axis similar to the Perelmann-Ricci Flow mapping, thus favoring formation of 2D type molecules. As represented by the toroidal topology [24] [25], this would then make the $\mathrm{z}$ axis of the toroidal an entangled variable between the $\mathrm{B}, \mathrm{D}$ Bosons and $\mathrm{a}$ quantum spectrum with

$$
E_{n}=\sum_{k=1}^{M} Q_{k} h v_{o} 2^{n+p} 3^{m}
$$

The factor 2 in Equation (8) comes from the $\tilde{\boldsymbol{P}}$ state while the power $n$ and $p$ correspond to $\mathrm{B}$ and $\mathrm{D}$ Bosons multiplicity. The factor 3 represents the 3 space variables and the power $\mathrm{m}$ is the quantum multiplicity.

And with Carbon-nitrogen molecules creating the nitrogenous bases. It is the thermal averaged excitation energy from that and the allowed quantum tunneling of the B, D DLRO bosons along an RNA or in a closed loop in a DNA. Thus we have the $\mathrm{B}$ and $\mathrm{D}$ eigenfunctions representing the genome spectra.

To understand Equation (8) let us consider a semi-conductor band, with an empty CB band separated to the filled VB band by a positive band gap. An electron can be excited from the VB into the CB. As this happens, the Coulomb binding will create an exciton, which is obviously a Bose-Einstein condensed Boson, as its center of mass has no translation motion, with quantum energy orbital levels within the band gap. These orbital levels depend on the effective masses of the electron and the hole, a property due to the crystal lattice structure. Suppose, we imposed a toroidal boundary, then it is obvious that both the excited electron and the hole closed orbits must wind around the toroidal. Pictorially we can compare such an orbital to winding a rubber band around a metal 
wire, then band the wire into a closed toroidal, which then gives us the expression for Equation (8). Note that each winding around the wire is of only a fraction of a quantum flux. The quantity $Q_{k}$ then represents the ensemble weight for the fundamental single winding with energy $h v_{o}$, which is a thermal averaged of its environment. The factor 3 thus represents the 3 orthonormal choices of the toroidal normal. The power $\mathrm{m}$ is then the stacking number of such toroidal.

\section{The Thermal Averaged Excitation Energy}

It is interesting to investigate Equation (4). And that all matters are then the result of thermal excitation $E^{\prime}$ from it.

We can look at the minimization of the thermal averaged excitation energy $\left\langle E^{\prime}\right\rangle$ w.r.t. $E_{o}$ or w.r.t. $T$. This optimization procedure is for obtaining the upper limit of $j$, as the lower limit is fixed at 1.

In the first case, we get

$$
\sum_{j=1}^{\infty}\left\{\frac{2}{j}-\frac{E_{o}}{k T}\right\} \mathrm{e}^{-E_{o} j / k T}
$$

The coefficient depending on $j$, vanishes at $2 k T=j E_{o}$. And becomes positive as long as

$$
2 k T>j E_{o}
$$

However that implies $E_{o} / k T$ must be quite small for $\left\langle E^{\prime}\right\rangle$ to contain a few terms in $j$.

If $E_{o}$ is $2 m(e) c^{2}$, the rest energy of two electrons, then with the lowest optimum $j=2^{5}=32$ gives us the rest energy of the bare Quark. $2^{5}$ represents $2 \times 2^{4}$, where the power 4 comes from the 4 homogeneous space variables, and the factor 2 comes from the B and D Bosons pair excitation. Fixing these numbers gives us the lowest $T$ value, which would be roughly $10^{7}-10^{8} \mathrm{~K}$, corresponding to the Bethe Fusion temperature.

Should we consider $E_{o}$ as the hydrogen binding R, which is $13.6 \mathrm{eV}$. Then we will get $T$ down to the range only of $400 \mathrm{~K}$ to $4000 \mathrm{~K}$. With Hydrogen at the lower $T$ end, and $j$ the number of stable elements matches the forming of the chemical elements in the periodic table at averaged temperature in the earth's crest. Note our arguments depend on assuming space homogeneity, or the masses formed from excitation of the Higgs vacuum are Poincare spheres. Thus this argument cannot be applied to the nitrogenous bases and hence life forms, as there the space is $2 \mathrm{D} \times 1 \mathrm{D}$. There we must refer back to the toroidal model, leading instead to Equation (8).

Now turning to minimizing $\left\langle E^{\prime}\right\rangle$ w.r.t. $T$, we get

$$
2 j E_{o}>[1+\sqrt{5}] k T
$$

This condition puts $E_{o}$ as an upper limit for the fundamental molecular coherent and de-coherent members, when $T$ is given at averaged earth surface temperature. This too is interesting because with either too high or too low $T$ values, 
these molecules might not be possible. A condition Schrodinger conjectured. Combining Equations (10) and (11) leads to

$$
4 k T>2 j E_{o}>[1+\sqrt{5}] k T
$$

Equation (11) puts a limit on $E_{o} / k T$

$$
4>2 j E_{o} / k T>[1+\sqrt{5}]
$$

Since $j$ is an integer starting from 1 , for the energy needed to create Quarks, $k T>64 m(e) c^{2}$. A result consistent with P1 projection for the creation of Quarks, at Bethe Fusion temperatures, so that equal number of protons to electrons can be simultaneously created to maintain the overall charge neutrality of matter as is required from starting with a net 0 charge $5 \mathrm{D}$ space-time universe. Therefore for the Higgs vacuum excitation to create matters, $k T$ must be much greater than $E_{\sigma}$.

Should $T$ be in the room temperature range, that is around $300 \mathrm{~K}$. which would be of order $10^{5}$ to $10^{6}$ lower than the Fusion temperature, the value of $E_{o}$ then can be down to $10-100 \mathrm{eV}$, which is in the atomic hydrogen binding range. Thus $T$ plays an important role in the formation of elements and molecules. The width of the $E_{o}$ range determines the number of stable elements and molecules as well as solid crystals that may exist at $T$. In fact, it automatically gives us the coherent and de-coherent molecular spectra. Thus $\mathrm{T}$ with its varying range plays a most important role in the types of stable matters that can exist. Our argument can be extended to life forms, where $\mathrm{j}$ optimum number than corresponds to its genome. Which in turn fixes the life form's body temperature. The more stable the $T$ value as needed for the specific life form, the better would be the DNA spectra to control the reproduction of proteins and cells in the body.

With this simple mathematical estimation, we show the importance of climate change on the survival of all life forms on earth.

As the B, D Bose-Einstein condensed state is excited by introducing $E_{o} / k T>0$, it simultaneously created a $4 \mathrm{D}$ non-homogeneous Lorentz manifold through a Perelmann-Ricci-flow mapping, thereby creating a toroidal structure, thus all energy eigen-solutions due to the kinematic of the masses contain within must satisfy the toroidal boundary. This condition was suggested by Frohlich in 1968 [26]. It is due to such imposed boundary condition, that led Geesink and Meijer [14] to come up with Equation (8).

Based on this formula and for a non-charged molecule, such as water, Geesink and Meijer [14] normalized its weight of 18 atomic mass unit as 1, and considered its spinning energy spectrum which must be proportional to its weight, satisfying Equation (8), they found that there exists two other atoms/molecules that will also be coherent. They were Carbon and Hydrogen molecule. The weight ratio of Carbon to water is $2 / 3$, while Hydrogen molecule to Water is $[1 / 3]^{2}$. Irrespective of charge and gauge invariance, the $5 \mathrm{D}$ to Lorentz $4 \mathrm{D}$ conformal mapping will result in a set of SU(3) generators, namely: $2 / 3,2 / 3$ and $-1 / 3$. Carbon being a covalent atom, therefore represents the two $2 / 3$ Lie group 
generators. For atomic weight there are no negative values. Thus for the $-1 / 3$, we can only have $[-1 / 3]^{2}$. It is therefore clearly verified that the fundamental set of coherent molecules obtained via the Perelmann-Ricci-flow mapping is water, carbon, and hydrogen. This essential result recognition shows the importance of these 3 elements as the key building blocks for more complex importance bio molecules, such as sugar, proteins and fats, and must be the building blocks in all life forms. Other coherent molecules consisted of other elements obviously can exist, but are less essential as they all satisfy higher products of the Lie group generators. A detailed list was provided in ref. [14]. Should we replace hydrogen in water by heavy hydrogen. That is changing the single proton to a proton and a neutron. This heavy water no longer satisfies the mass unit normalization, hence its spinning eigen-energy does not obey Equation (8), and its spectrum becomes Decoherence. The presence of heavy water is then detrimental to life forms. Of course heavy water is radioactive, and one might think this example is a not conclusive proof of Decoherence effect. Because some gases such as chorine and fluorine dissolve in water, their presence would essentially also change the non-pure water spinning eigen-energies into Decoherence, and hence detrimental to life forms. We use this result for disinfection of our swimming pool water, or in killing germs.

The coherent biomolecular spectra span from roughly $10 \mathrm{~Hz}$ to $500 \mathrm{~Hz}$. Because of the low energies, it contains more resolvable information than the higher frequencies, and actually coincide with our modern 5G telecommunication. In fact, this same spectra covers all the life forms DNA spectra and is used in the growth mechanism. Hence constant exposure to such external radiation source would be harmful to the proper growth of our body. Unless there is a technological way to prevent our body exposure to the $5 \mathrm{G}$ telecommunication sources it should not be used. On the other hand, realizing this coherence spectra can also be applied beneficially to induce our body to counter cancerous growth. One method is targeting the cancerous growth by applying a corrective radiation through the nerve system via the technique of acupuncture. Both of these topics certainly deserve further research. It was well-known that soothing music in this audible range helps our body and mind, while exposure to decoherent noise is harmful.

\section{Life Forms}

Although the coherent molecules such as water, hydrogen and carbon are essential building blocks for hydrocarbon bio matters, such as proteins, fats, muscles, bio-cells, etc., we still need a mechanism over and on top of energy to make them happen and grow so that we can get life forms. It was postulated by us [16] that this came about because of the matching of the DNA frequency spectra which are of DLRO as they are eigen-solutions of the B, D bosons to those of the coherent molecules, such that via formation of ODLRO in the bio-systems can such ordered matching occur. In another word, it is via a superconducting tran- 
sition that the coherent molecules were assembled to form the bio cells, proteins, fats, etc. and eventually the entire life form.

The superconductivity phase transition can occur if the environmental temperature $T$ is below the superconducting critical Temperature Tc. As such the life form's body temperature $\mathrm{T}$ must remain below the Tc's for each and every bio cell, and bio-structures within its body. But since the coherent molecules building blocks are charge neutral, all bio-structures, its electronic structure can only be semi-conductor like, with a partially filled valence band and an empty conduction band separated from the valence band with a positive band gap G. Such a band structure resembles that of the known High Temperature Cuprate Superconductors [27] [28] [29]. Hence, the bio-growth mechanism is the same as that in the ceramic HTC. The DNA of a life form is composed by the stacking of two linear chain of side by side nitrogenous bases. Within each nitrogenous base, there are three side by side carbons, and then separated with a nitrogen on both ends, before attaching to a backbone carbon twisting chain. There are in fact 3 such distinct bases that permit the B, D bosons that might exist in the side by side carbon nuclear time frozen $5 \mathrm{D}$ cores to quantum tunnel to the adjacent level base, while there is one end cap nitrogenous base, that only allows for reflection. Hence, a closed loop of the B, D DLRO can be formed. Because the B, D are DLRO bosons, that are in fact the magnetic monopoles, their quantum flux unit is governed by a $\pi$ rotation [2] instead of $2 \pi$, the 3 adjacent carbons within each nitrogenous base then exactly satisfy this gauge requirement. It is this topological confinement and the $3 \mathrm{D}$ space dimension projection into $2 \mathrm{D} \times 1 \mathrm{D}$ that must happen according to the $5 \mathrm{D}$ symmetry breaking we are led to the formation of these 4 nitrogenous base structures. Because of such features of the $4 \mathrm{ni}$ trogenous bases, there are different stacking orders possible in the formation of the DNA of a life form. It is such distinct stacking orders that give the genome. Since each specific order stacking within the DNA contains a unique DLRO spectrum, it would induce a similarly order the choice of coherent molecules via a ODLRO phase transition of the VB holes to form the specific bio cell and its Point Group structures. Hence, we postulate that life forms are also a natural result from the 5D homogeneous symmetry breaking. Starting with simple viruses, such as COVID-19 [30] [31], to the most complex known for us human [16]. But because its existence is subjected not only to the Bose distribution excitation of the $\mathrm{B}, \mathrm{D}$ bosons, but also the two key temperature requirements. First that from the liquid phase of water, and second the bio body temperature $\mathrm{T}<$ Tc's of all the bio-cells and bio-materials within the body of the life form. Any changes to the temperature would lead to sickness or death of the life. In short, starting with the 5D universe, each successive step of creation appears to follow a determined sequence, all the way to the creation of us human.

\section{Conclusions}

Since the publication of the book "The Five Dimension Space-Time Universe. A 
creation and grand unified field theory model", we had published several detailed articles covering numerical hadron mass calculations, to astronomical data of stars and planets, as well as the explicit Maxwell's magnetic monopoles, based on several mathematical theorems, starting with Fermat's Last Theorem [32] to Perelmann's Ricci-Flow and entropy mappings, and the resolving of the SU(2) and SU(3) Lie Groups as a direct product to the Poincare Group as required by the McGlinn's Theorem. Thus verifying the Lee-Yang theory [33] on the Parity violation in Beta Decays [34], and the Gell-Mann Standard Model for hadrons. From these, we had also extended to the fully relativistic $2 \mathrm{D}$ hydrogen model with the exact 2D Chern-Simons gauge [35]. Less rigorous extensions were also proposed by us for the formation of the nitrogenous bases in RNAs and DNAs, thus leading to our modeling for life forms, from human to COVID. Then starting with the explicit monopoles, we show its mathematical equivalence to the Higgs Bosons. Through that established a Higgs vacuum in the time irreversible ever expanding 5D Universe. Each step of our investigation led to other branches of scientific researches. Including the ideas of Schrodinger that Life is a fully quantum phenomena [26]. It is to all these efforts that this current paper also aims. In particular in this current paper, we try and connect the Florhlich's idea of the coherence quantum vibration sequence in biomolecules, thus justifying the Geesink-Meijer formula for the coherence biomolecules. Perhaps of most interest is it did lead to the identification of water, carbon and hydrogen as the essential building blocks of all bio-materials [36]. Last but not least, we were able to connect the Einstein's Cosmo Constant in the Gravity tensor equation to that of the energy density derived from the thermal excitation of the Higgs vacuum, a result due to entropy as postulated in the Perelmann's mappings. In this current paper, the Dirac second quantization representation, only serves as a more pictorial explanation of the physics we have previously found, itself does not give us new knowledge. Yet, indeed there remain many more detailed questions for us to investigate. Including details to each bio cell's relationship to the DNA and the body's functions, and methods of RNA, DNA modifications, and its potential in treatment of inherited deceases. [see for example the viro-vector approach in modifying the RNA thus leading to a non-defective DNA for certain rare childhood cancer therapy of Rocket Pharma, RCKT].

So far, we, human have achieved all these because we are the only life form on earth born with a brain capable of sophisticated intelligence, and able to accumulate knowledge through learning and logical analysis. Today we even can modify our own DNAs as we mentioned by RNAi methods. Recently through mRNA method, Pfizer developed a vaccine for the COVID-19 pandemic. As well as biotechnology firms similar to Rocket, through RNAi developed cure for some cancers. However, human is still like all other life forms, he possesses a natural selfish preservation instinct. It is this self-preservation instinct that different races and nations foster racism, and occupation of weaker nations through wars. Today, human technologies have created self-destructive weapons that can 
easily lead to its own extinction, just like other animal species in history. For human to survive, it must change its behavior, and promote LOVE, love of all nature's creations, race equality, and global cooperation among nations, if we, human are to be able to succeed to understand fully all of Creation, and the Creator's purpose. Despite the title of this section being called "Conclusions", I would suggest that it should be more appropriately named as an introduction to the effort for understanding the Creator's true purpose and for our own existence.

\section{Acknowledgements}

We thank Ms. Winnie So for her help in the manuscript, and Professors G. Dreschhoff, H. Jungner and P.C.W. Fung for their contributions in much of the cited previous publications in particular the Five Dimension Space-Time Universe Book, published by Scientific Research Publishing, USA in 2014, without which this summary note with second quantized Dirac Representation cannot be written.

\section{Conflicts of Interest}

The authors declare no conflicts of interest regarding the publication of this paper.

\section{References}

[1] Wong, K.W., Dreschhoff, G.A.M. and Jungner, H. (2014) The Five Dimension Space-Time Universe. A Creation and Grand Unified Field Theory Model. Scientific Research Publishing, USA.

[2] Perelmann, G. (2002) The Entropy Formula for the Rick Flow and Its Geometric Applications.

[3] Perelmann, G. (2003) Ricci Flow with Surgery on Three-Manifolds.

[4] Kaluza, T. (1921) ZumUnitätsproblem der Physik, Sitzungsbericht der K. Preussischen Akademie der Wissenschaften. MathematischePhysik, Berlin, 966.

[5] McGlinn, W.D. (1964) Physical Review Letters, 12, 467. https://doi.org/10.1103/PhysRevLett.12.467

[6] Gell-Mann, M. (1964) Physical Review Letters, 12, 155. https://doi.org/10.1103/PhysRevLett.12.155

[7] Wong, K.W., Dreschhoff, G. and Jungner, H. (2012) Journal of Modern Physics, 3, 1450-1457. https://doi.org/10.4236/jmp.2012.310179

[8] Maxwell, J.C. (1864) Philosophical Transactions of the Royal Society, 155, 459-512.

[9] Wong, K.W., Dreschhoff, G., Jungner, H., Fung, P.C.W. and Chow, W.K. (2018) Physics Essays, 31, 493-495. https://doi.org/10.4006/0836-1398-31.4.493

[10] Higgs, P.W. (1964) Physical Review Letters, 13, 508-510. https://doi.org/10.1103/PhysRevLett.13.508

[11] The CMS Collaboration (2012) Science, 338, 1569-1575. https://doi.org/10.1126/science.1230816

[12] Einstein, A. (1916) Annalen der Physik, 49, 769-822. 
https://doi.org/10.1002/andp.19163540702

[13] Toms, D.J. (1984) An Introduction to Kaluza-Klein Theories. In: Workshop on Kaluza-Klein Theories, Chalk River/Deep River, World Scientific Publication, Singapore, 185.

[14] Geesink, H.J.H. and Meijer, D.K.F. (2018) Journal of Modern Physics, 9, 898-924. https://doi.org/10.4236/jmp.2018.95056

[15] Streltsov, A., Adesso, G. and Plenio, M.B. (2017) Review of Modern Physics, 89, Article ID: 041003. https://doi.org/10.1103/RevModPhys.89.041003

[16] Wong, K.W., Fung, P.C.W. and Chow, W.K. (2019) Journal of Modern Physics, 10, 1548-1565. https://doi.org/10.4236/jmp.2019.1013103

[17] Liu, J., Zhang, H., Howard, A.W., et al. (2019) Nature, 575, 618-621. https://doi.org/10.1038/s41586-019-1766-2

[18] Abdul-Masih, M., Banyard, G., Bodensteiner, J., et al. (2020) Nature, 580, E11-E15. https://doi.org/10.1038/s41586-020-2216-X

[19] Wong, K.W., Fung, P.C.W. and Chow, W.K. (2019) Journal of Modern Physics, 10, 557-575. https://doi.org/10.4236/jmp.2019.105039

[20] Wheeler, J.A. (1955) Physical Review, 97, 511-536. https://doi.org/10.1103/PhysRev.97.511

[21] Wong, K.W. and Chow, W.K. (2020) Journal of Modern Physics, 11, 1911-1917. https://doi.org/10.4236/jmp.2020.1112119

[22] Fung, P.C.W. and Wong, K.W. (2015) Journal of Modern Physics, 6, 2303-2341. https://doi.org/10.4236/jmp.2015.615235

[23] Fung, P.C.W. and Wong, K.W. (2017) Journal of Modern Physics, 8, 668-746. https://doi.org/10.4236/jmp.2017.84045

[24] Lu, L., Xia, L., Chen, Z., et al. (2020) NPJ Quantum Information, 6, 30. https://doi.org/10.1038/s41534-020-0260-X

[25] Geesink, H.J. (2020) Energy States of Entangled Semiconductors Described by a Toroidal Code and a "Balanced Signal Technology".

[26] Frohlich, H. (1968) International Journal of Quantum Chemistry, 2, 641-649. https://doi.org/10.1002/qua.560020505

[27] Ching, W.Y., Xu, Y.N., Zhao, G.L., Wong, K.W. and Zandiehnadem, F. (1987) Physical Review Letters, 59, 1333. https://doi.org/10.1103/PhysRevLett.59.1333

[28] Wong, K.W. and Ching, W.Y. (2004) Physica C, 416, 47. https://doi.org/10.1016/j.physc.2004.09.003

[29] Wong, K.W. and Curatolo, S. (2008) EEM: The Exciton Enhancement Mechanism Theory and Experimental Evidence of Optically Enhanced Tc in High Tc Superconductors. In: Chang, O.A., Ed., Progress in Superconductivity Research, Nova Science Publishers, Inc., Hauppauge, 55-78.

[30] Wong, K.W., Fung, P.C.W. and Chow, W.K. (2020) Open Journal of Biophysics, 10, 88-95. https://doi.org/10.4236/ojbiphy.2020.102008

[31] Matsuyama, E. (2020) Journal of Biomedical Science and Engineering, 13, 140-152. https://doi.org/10.4236/jbise.2020.137014

[32] Aczel, A.D. (1997) Fermat's Last Theorem: Unlocking the Secret of an Ancient Mathematical Problem. Penguin Press, London.

[33] Lee, T.D. and Yang, C.N. (1956) Physical Review, 104, 254. https://doi.org/10.1103/PhysRev.104.254 
[34] Wu, C.S., Ambler, E., Hayward, R.W., Hoppes, D.D. and Hudson, R.P. (1957) Physical Review, 105, 1413-1415. https://doi.org/10.1103/PhysRev.105.1413

[35] Gou, S.H., Yang, X.L., Chan, F.T., Wong, K.W. and Ching, W.Y. (1991) Physical Reviews A, 43, 1197-1205. https://doi.org/10.1103/PhysRevA.43.1197

[36] Geesink, H.J. (2020) Proposed Informational Code of Bio Molecules and Its Building Blocks: Quantum Coherence versus Decoherence. 